\title{
Performance Trade-Offs for Wavelength Striping Optical Switching Using a Novel Star Architecture
}

\author{
Enrique Rodriguez-Colina, Michael Pascoe-Chalke, and Miguel Lopez-Guerrero \\ Department of Electrical Engineering, Metropolitan Autonomous University (UAM-I), 09340 Iztapalapa, DF, Mexico \\ Correspondence should be addressed to Miguel Lopez-Guerrero; milo@xanum.uam.mx
}

Received 31 October 2015; Revised 11 January 2016; Accepted 13 January 2016

Academic Editor: Michael A. Fiddy

Copyright ( 2016 Enrique Rodriguez-Colina et al. This is an open access article distributed under the Creative Commons Attribution License, which permits unrestricted use, distribution, and reproduction in any medium, provided the original work is properly cited.

\begin{abstract}
This work describes various performance trade-offs that arise from the use of a technique for optical switching under various network topologies. Such switching operation can be summarized as follows: (a) user data are divided into fixed-length fragments, (b) each fragment is assigned to a different wavelength, and (c) all wavelengths are simultaneously switched to the egress links. This concept of dividing user data into several wavelengths to be simultaneously switched is called wavelength striping and its purpose is to reduce latency and increase throughput for short distance interconnects. We depart from previous work where a building block implementing this basic switching function has been built around semiconductor optical amplifiers (SOAs). In this paper, we investigate diverse trade-offs that arise from the use of this switching approach in different network topologies. One of the main issues addressed in this paper is the relation between cascadability and bit error rate (BER). In this case, our results indicate that a switch fabric can cascade up to five stages without exceeding a BER of $10^{-9}$ and without incurring in power budget problems. We also show that the performance degradation, introduced by cascading SOAs, can be compensated with a star interconnect architecture that is introduced. Other issues addressed in this paper are the effect of scalability on cost and the effect of latency on TCP performance and reliability.
\end{abstract}

\section{Introduction}

All-optical signal processing has already been recognized as a necessary step in the evolution of broadband networks (see, e.g., [1]). In this context, the performance of optical communication infrastructure depends, up to a large extent, on the underlying switching technology. For instance, solutions based on wavelength division multiplexing (WDM) incur in relatively long latencies during the switching process. This is due to the fact that the switch must wait until enough data is accommodated in a single wavelength before the switching action takes place. An alternative to WDM is the use of wavelength striping also known as bit per wavelength WDM (BPW) [2]. This technology reduces switching latencies due to the simultaneous forwarding of short stripes of data in parallel. This strategy has the additional advantage that when data naturally occur in parallel (e.g., the data bus of a microprocessor), no parallel-to-serial conversion is needed since parallel pulses can be simultaneously launched on different wavelengths [2]. This concept of using various wavelengths to achieve simultaneous transmissions of bits or packet fragments per time slot is referred to as wavelength striping.

Although traditional topologies such as bus, ring, and star have been widely studied for networks based on electronics and wavelength division multiplexing [3-5], studies considering the wavelength striping approach in optical interconnects are rather scarce (see, e.g., [6-8]). This field requires more topological research since there are relevant differences between the approaches of electronics and photonics that prevent us from extrapolating results from one domain to the other. Such differences include, for example, the possibility of cheaper signal regeneration in electronics than in optical devices and, as a consequence, electronics facilitate the cascading of more devices. Electronics also makes it possible to buffer data and to use random access memory. Optics, 
however, can offer more bandwidth and higher immunity to electromagnetic interference [9]. Regarding the differences between WDM and wavelength striping, it can be mentioned that the intended applications for the latter are the ones where a combination of high bandwidth and low latency is required. This characteristic becomes relevant in protocols that are sensitive to the bandwidth-delay product (e.g., TCP [10]). Wavelength striping is recommended for links where transmission times are significantly longer than propagation times, for example, short-reach interconnects. Examples of networks that could be suitable for wavelength striping are electronic circuit boards and room-sized networks. It can also be used in applications such as storage area networks (SANs) with optical interconnects or optical switches with short distances between nodes. Other possible applications include database-intensive applications, parallel data processing, and video graphics.

Cascadability is an important concept related to the topology of optical interconnects. The cascadability limit is defined as the maximum number of devices that can be connected in series, so that the quality of the signal is maintained within adequate levels in order to receive coherent data. In this work, we study the trade-off between bit error rate (BER) and cascadability of topological variants of wavelength-striped optical interconnects using semiconductor optical amplifiers (SOAs). To this end, we report our findings from studies of cascadability for a switching system using photonic devices. Our experiments suggest that the maximum number of SOAs that can be cascaded is limited to five devices in order to achieve a BER less than $10^{-9}$. This finding is used in order to investigate the topological limit of the Photonics Layer.

Another relevant concept addressed in this work is scalability, which is defined as the total number of outputs, or ports, of the system. We investigate the scaling properties of diverse network topologies since this concept has a direct effect on the way costs increase when connecting an increasing number of network nodes. Other aspects brought about by the network topology such as reliability, latency, and TCP performance are also analyzed.

The remainder of this paper is organized as follows. Section 2 describes the architecture of the switching device used in the implementation of the network topologies. Section 3 introduces the three analyzed topologies. Section 4 describes the assumptions and considerations for the SOA model and for the analysis. Section 5 presents the results and analysis of the topologies. Section 6 concludes this work.

\section{Basic Switching System Architecture}

In the system under consideration, different wavelengths are used to transmit data in parallel between pairs of computers, as shown in Figure 1. In order to establish the communication link, an electrooptical switch fabric is utilized, but without signal conversion from optics to electronics in middle stages. At the edges, that is, computers at the ends, such conversion is carried out.

Since wavelength striping is used, data from the same user are distributed over different wavelengths. As depicted in Figure 1, data arriving to the switch fabric are stripped using stripes of fixed length. Each data fragment is assigned to a different wavelength and, in each time slot, all wavelengths are simultaneously switched. Note that the time slot and the stripe size are closely related. This is due to the fact that the number of bits that can be allocated in the stripe is restricted by the slot duration. The slot size is adjusted by taking into consideration the desired size of the stripes plus a gap used as a guard band. It is worth pointing out that the stripe size could be as short as one bit; however, the process required to insert a single bit into a time slot requires a complex electronic control to perform very high speed switching. As a consequence, this approach becomes impractical. On the other hand, if the stripe size is excessively long, the advantage of fast switching is compromised and the system may not have any advantage over WDM. Consequently, the optimum stripe size depends on the control characteristics and the requirements of both the switch fabric and the applications.

An SOA-based architecture for a wavelength striping switching system without cascaded interconnects (i.e., only one stage interconnecting two end devices) has been presented in $[11,12]$. These studies were developed for the SWIFT/SOAPS demonstrator project. Among the major features of this setup, it is worth mentioning that the medium access control (MAC) was implemented using field programmable gate array (FPGA) boards. These devices control the time slots, the interaction among nodes, and the scheduling of tasks and they also implement the central "arbiter," which is part of the control system. The electronic arbiter is in charge of switching the SOAs on and off and its control signal is distributed using an independent control channel over an out-of-band wavelength.

Among other relevant characteristics of this system, it can be mentioned that the use of idle tones, when data are not being transmitted, avoids transients in the switches and facilitates the use of coupled transceivers. The receiver in the short-reach network is semisynchronous, in the sense that although there is packet-level synchronization, there is no synchronization at the bit level. The received signal characteristics allow the receiver node to perform clock recovery from it. A time slot lasts $2.83 \mu$ s and there is a guard band of about $0.5 \mu$ s between time slots, which allows for a maximum utilization of $85 \%$.

The typical sequence of actions that takes place during a transmission is as follows. The host that is about to send data must transmit a request for a time slot to the arbiter. In consequence, the arbiter allocates a time slot and passes on the slot allocation information to the host, which, in turn, prepares itself to launch the data within the allocated time slot. The payload, that is, the bits that carry the data generated by the host, is always preceded by a (no signal) guard band which allows the switch to be fully configured by the arbiter by the beginning of every time slot. The medium access control is therefore based on a request-grant exchange between the host and the arbiter.

A number of experiments were carried out on a real implementation of the switching architecture just described (see Section 4). Data resulting from these tests were used to calibrate the simulation model used to study the following 


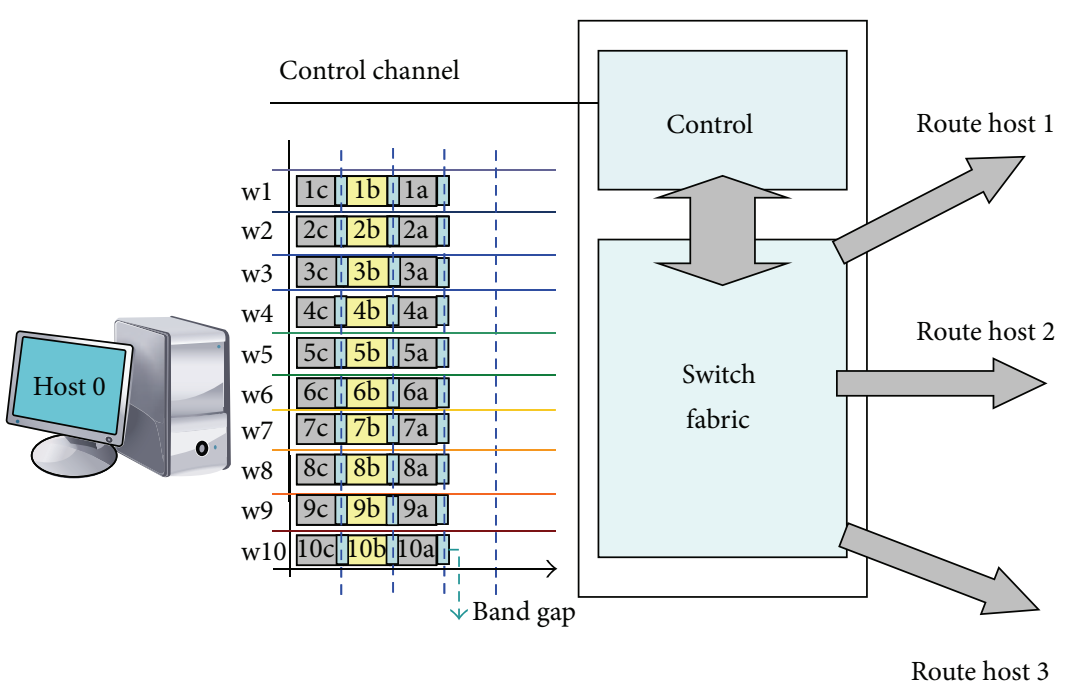

Switching time $=$ time slot

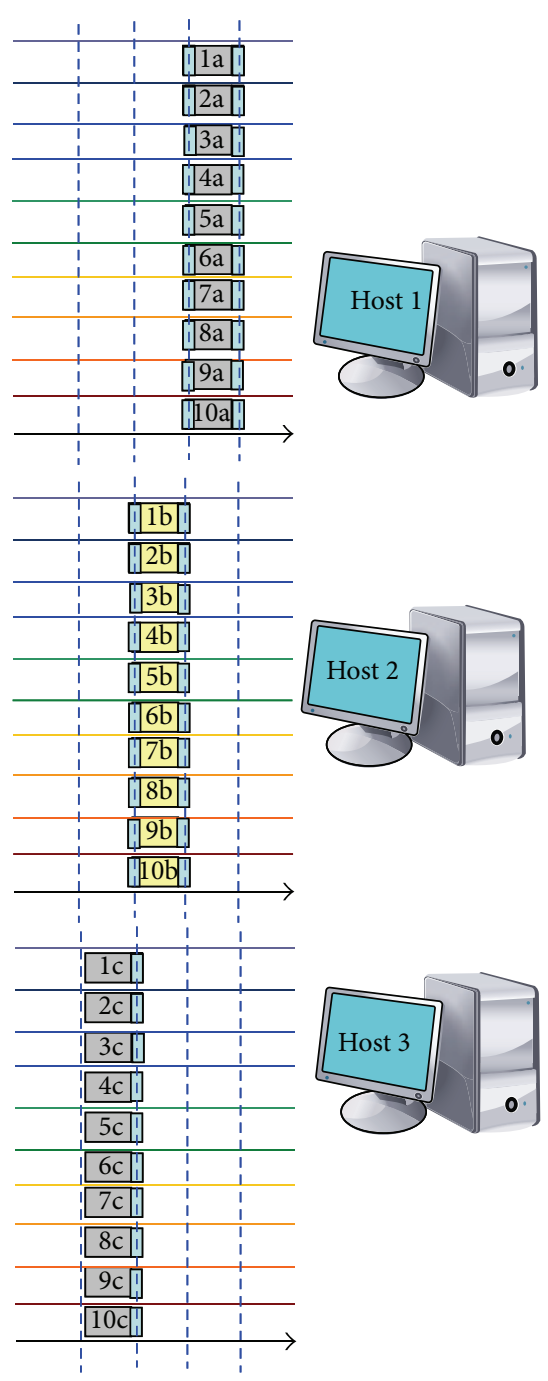

FIGURE 1: A ten-wavelength striping system with semisynchronous time slots.

network topologies for the switch fabric: tree, ring, and star. These topologies are described in the following section.

\section{Tree, Ring, and Star: SOA Interconnect Topologies}

In this section, we describe the topologies that will be considered in the analysis with emphasis on the way they affect the carrier signal. Network topology also has an effect on scaling since, depending on the way network elements are interconnected, an increase in the number of network nodes requires a different increase in the number of couplers and SOAs. Therefore, the choice of the topology in the design of optical interconnects is critical, since it affects both BER performance and implementation costs.

3.1. Tree Topology. In the tree topology (i.e., bus), the main trunk is successively split to form branches. As illustrated in Figure 2, different paths may continue in sequence with the same branching structure. It is possible to switch between separate paths using two SOAs connected to the outputs of a splitter, with 1:1 splitting ratio. The SOAs can be switched on and off by the electronic control module in order to establish the path from the source to the destination. In this case, one SOA will transmit light and the other one will block it when the former is switched on and the latter is switched off. Both could be switched on for broadcast purposes.

It is worth emphasizing that cascadability in tree topologies is the linear addition of paths. On the other hand, scalability equals the total number of branches, that is, the number of nodes that can be reached by a single transmission. The achievable cascadability comes from establishing upper limits in power losses, signal deterioration, interference, noise, and dispersion.

In this work, we consider that the tree is created without signal regeneration (i.e., it is built with passive components only), because the interest is in transmitting the optical signal without conversion to electronics, and without the use of buffering in middle stages. 


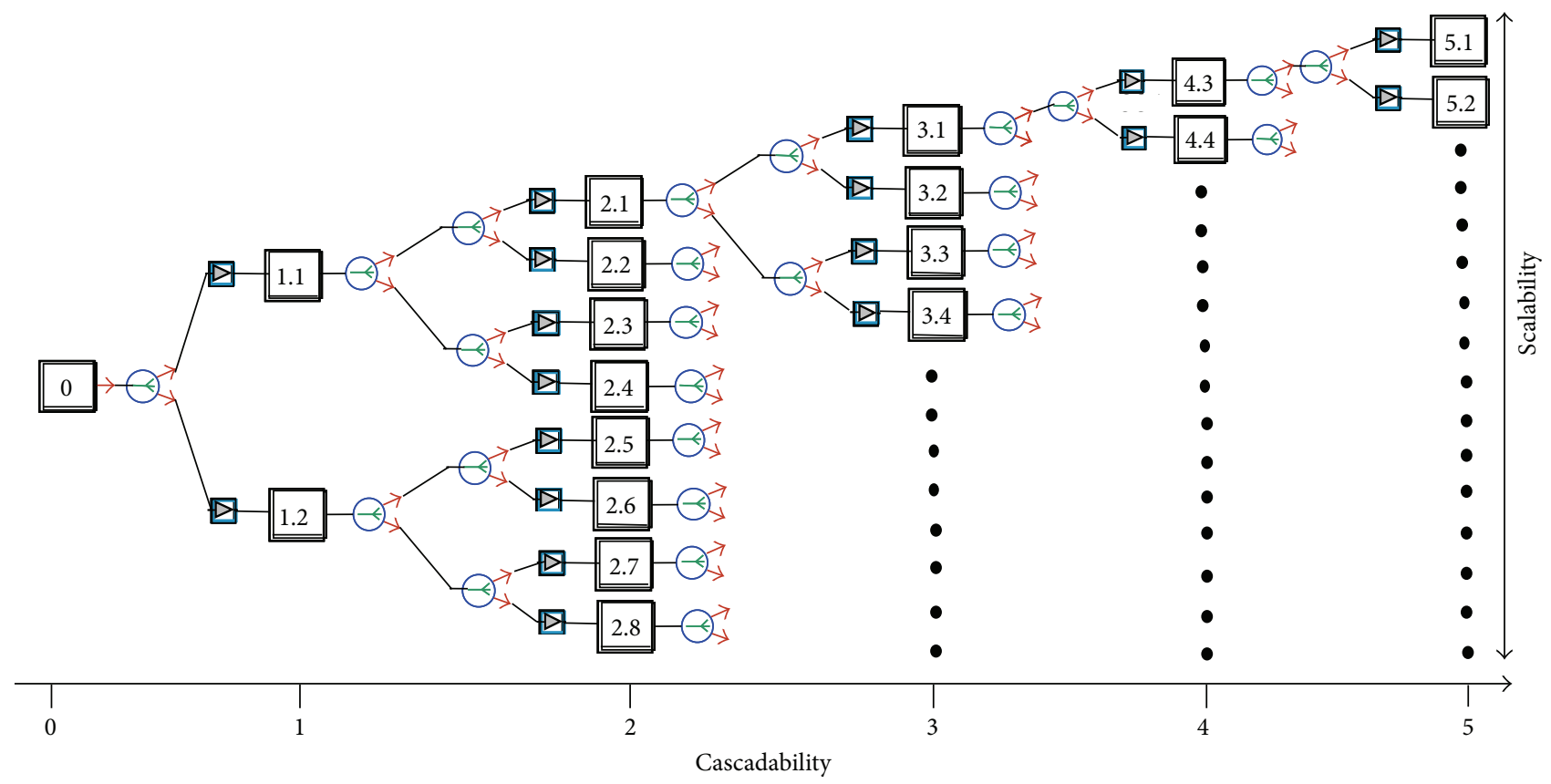

FIGURE 2: SOA interconnection in tree topology.

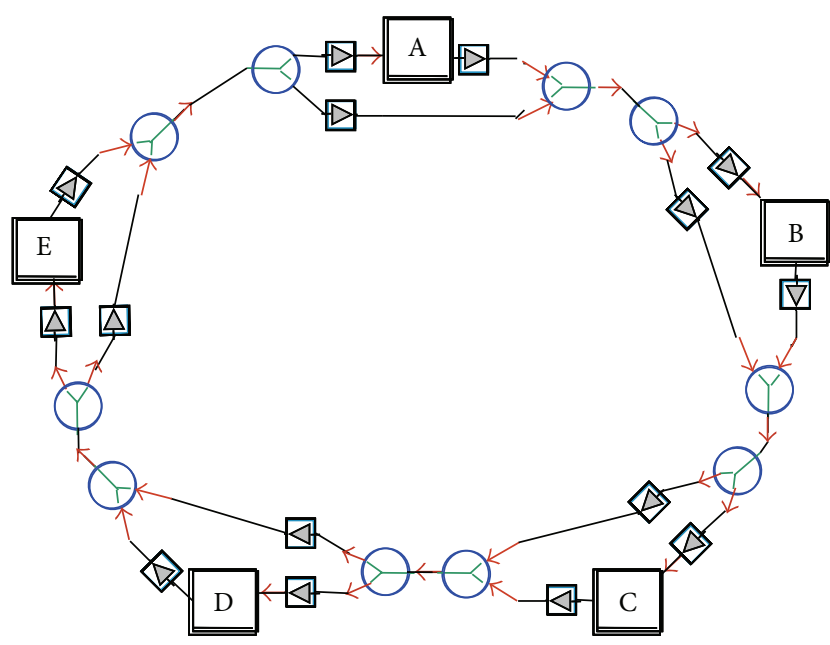

FIGURE 3: SOA switch interconnection in ring topology.

3.2. Ring Topology. The ring topology is formed by nodes connected one after the other creating a closed loop. In fact, Tanenbaum [13] describes the ring topology as a collection of point-to-point links where nodes may be connected using a T-junction. Figure 3 shows a ring structure based on SOA interconnections. As illustrated in this figure, in the case of rings with passive interfaces, a node disconnected from the ring does not affect the network, only the node itself. This is due to the presence of an alternative link, that is, a bypass link, at each node that connects the next node in the ring. One of the drawbacks of this topology is the loss of light at each junction due to the directional couplers, which results in reduced cascadability when nodes are added. In this case, signal regeneration can be incorporated at the nodes [14], which allows an increased number of stations or nodes to be cascaded in the ring as compared to the case without regeneration. A ring with regeneration or active repeaters has cascadability advantages, but it has the disadvantage of a high cost due to the use of expensive components and the need of electrooptical conversion. Due to these reasons, only the ring topology without signal regeneration is considered in this work.

3.3. Star Topology. In this subsection, we introduce SOAbased star topology for wavelength striping optical switching. The proposed star topology is created by using SOAs to connect the network nodes to the inputs and outputs of a multiport coupler or central distributor as shown in Figure 4(a). The central distributor implements structures with nonblocking paths which fully interconnect all nodes (see Figure 4(b)).

In broadcast communications, the source node transmits to the central distributor and then the signal is forwarded to all its output ports. In this case, the energy at the output is divided among the outgoing lines.

In point-to-point mode, a receiver selection process is executed in order to route the data to the proper destination node. For the selection process, the electronic control enables the corresponding path by switching on the SOA in the destination node, whereas the rest of the SOAs are switched off. Thus, this topology has the advantage of facilitating the selection of only the nodes that need to be connected. This means that communication between two nodes does not have to pass through other nodes, as it is the case in the ring topology. This gives the advantage of fewer cascaded SOAs for the star topology. In this case, the distributor requires a more complex and expensive system since the star centralizes all 


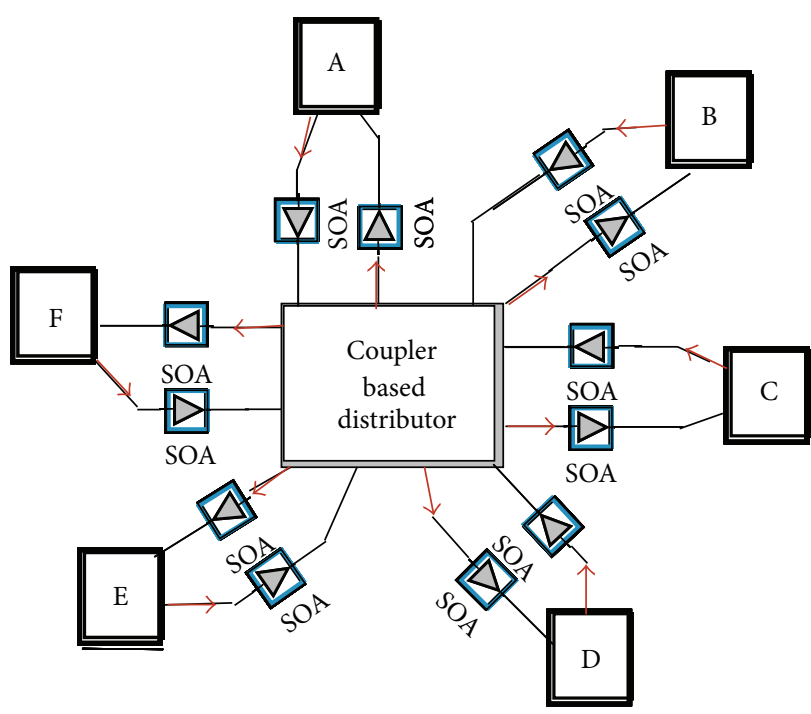

(a)

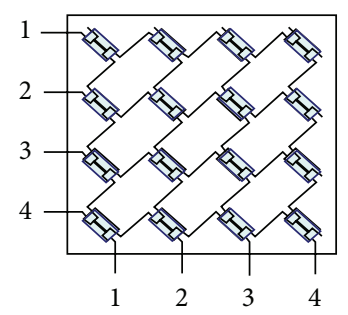

(b)

FIGURE 4: (a) Star topology and (b) example of a central distributor based on $2 \times 2$ couplers for a 4-node star.

the connections and the central distributor adds propagation delays and splitting losses (in the multiport couplers, the distribution loss is related to the coupling loss). A drawback of this topology is that if the central distributor fails, then the entire system is affected.

\section{Considerations for the Performance Study}

Several issues have to be taken into consideration for the system evaluation. The most relevant ones are described in this section.

4.1. Cross-Gain Modulation Effects. Data transmission is affected by the number of aggregate wavelengths. This is due to the fact that modulated signals passing through an SOA with multiple wavelengths are susceptible to saturation in optical devices [15]. This is caused by the aggregation of input power which generates changes in the carrier density and, therefore, produces a cross-gain modulation effect. This cross-gain modulation effect causes cross talk which leads to degradation of the overall system performance. The power in each wavelength $\left(P_{\lambda i}\right)$ affects the carrier density $(n)$ of the other wavelengths that are injected into the SOA. This effect can be studied by using the following rate equation [16-18]:

$$
\frac{d n}{d t}=-V_{g}\left(n-n_{0}\right) \sum_{i=1}^{M} P_{\lambda i}
$$

where $M$ is the number of wavelengths in the system, $V_{g}$ is the group velocity, and $n_{0}$ is the carrier density required for transparency. Thus, the variation in a carrier signal depends on the power of the wavelengths. Thus, the SOAs in the switch fabric are susceptible to changes in the input power which may selectively affect the wavelengths.

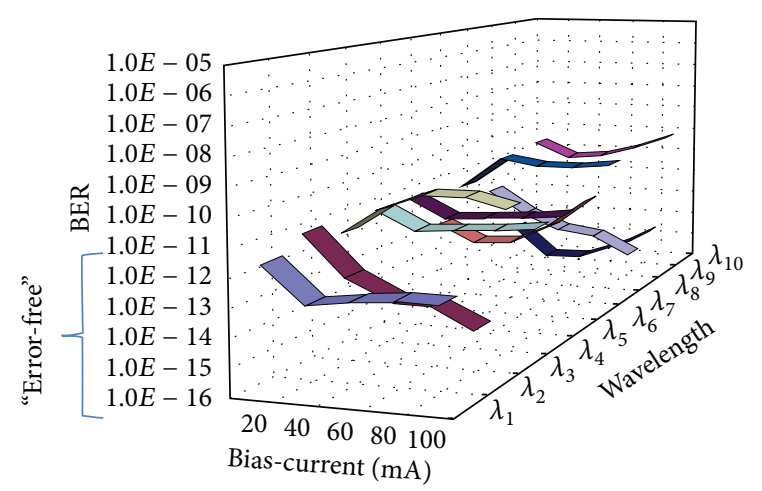

FIGURE 5: BER versus bias-current for each one of the ten wavelengths, single SOA model.

4.2. Q Factor and BER. We performed some experiments to assess the level of signal degradation as a function of the bias-current (i.e., gain) of the SOA. When the gain increases, the SOA reaches a saturation point and this induces signal distortion which decreases the $Q$ factor and therefore increases the BER. In addition, BER differences among different wavelengths are also expected due to random variations in the input power of each wavelength; thus, these variations have to be included in the simulation model. This is a necessary factor to be taken into account in order to perform reliable simulations of a real device where the input power to the SOA is slightly different for each wavelength source. The software used in this work (i.e., VPI [16]) allows the introduction of these random variations in the simulations.

Figure 5 illustrates the simulation results of BER as a function of the bias-current of a single SOA in a ten-wavelength setup. The wavelengths were uniformly distributed between $\lambda_{1}=1550.71 \mathrm{~nm}$ and $\lambda_{10}=1554.33 \mathrm{~nm}$ and spaced $0.4 \mathrm{~nm}$ apart $(50 \mathrm{GHz})$. These results were obtained when the bias-current was changed in an interval between $20 \mathrm{~mA}$ and 


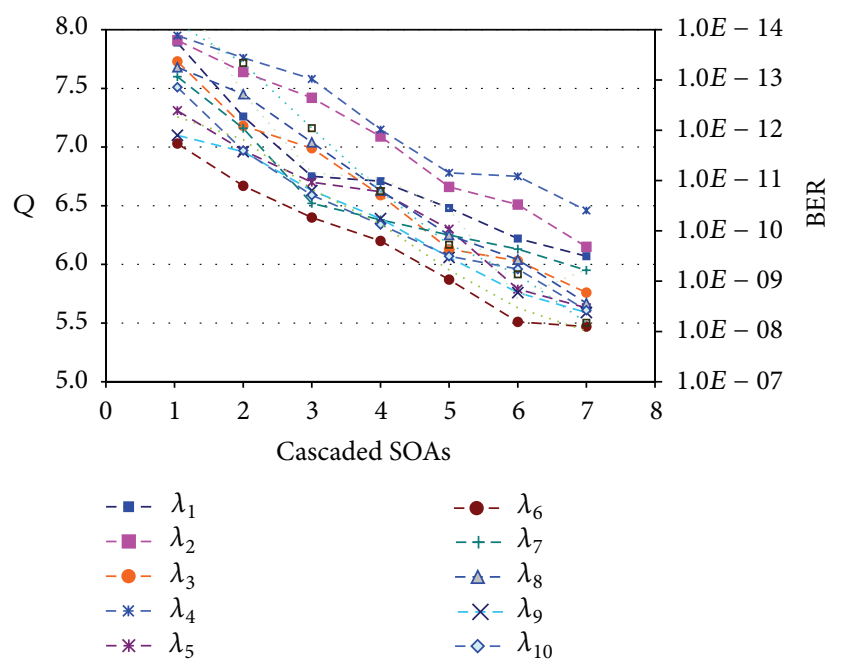

FIGURE 6: $Q$ factor and BER versus cascaded SOA stages with ten wavelengths.

$100 \mathrm{~mA}$. As expected, it is shown that the BER is different for each wavelength considered in the experiment. Nevertheless, most figures are within an average range considered as "errorfree" transmission (i.e., BER figures lower than $10^{-12}$ ) for main standard telecommunication requirements.

We also simulated the interconnection of SOAs in series in order to investigate the way the number of cascaded SOAs affects the $Q$ factor. The simulation results are depicted in Figure 6.

Unless the signal is regenerated when cascading, signal distortion produces a detrimental effect on the $Q$ factor. In laboratory test-beds, systems without signal regeneration showed a similar distortion of the signal. This implies that both correct coupling and power balancing between stages are required to optimize the $Q$ factor.

4.3. Cascadability Limit. In order to add node connections with nonblocking routing in optical short-reach SOA-based switches, it is necessary to cascade SOA stages. The disadvantage, however, is that cascadability distorts the transmitted signal and, as a consequence, the BER increases.

In order to find out the limit in the number of SOAs that can be cascaded without significant signal degradation, we conducted computer simulations. The results are shown in Figure 7 (these results are related to the ones in Figure 6), where the BER is plotted against the number of cascaded SOAs. Each dot in Figure 7 represents the average BER for 10 -wavelength signals crossing the SOAs. It can be observed that the trend follows an exponential increase, which is represented with a broken line. These results indicate that up to five stages can be cascaded in practical interconnect applications maintaining a BER less than $10^{-9}$.

Figure 8 depicts the distortion that can be observed as more cascaded SOAs are added to an optical data path. The reduction in the aperture of the eye diagrams suggests that the BER increases after each cascaded SOA. The eye diagrams show that the opening of the eye is reduced both horizontally

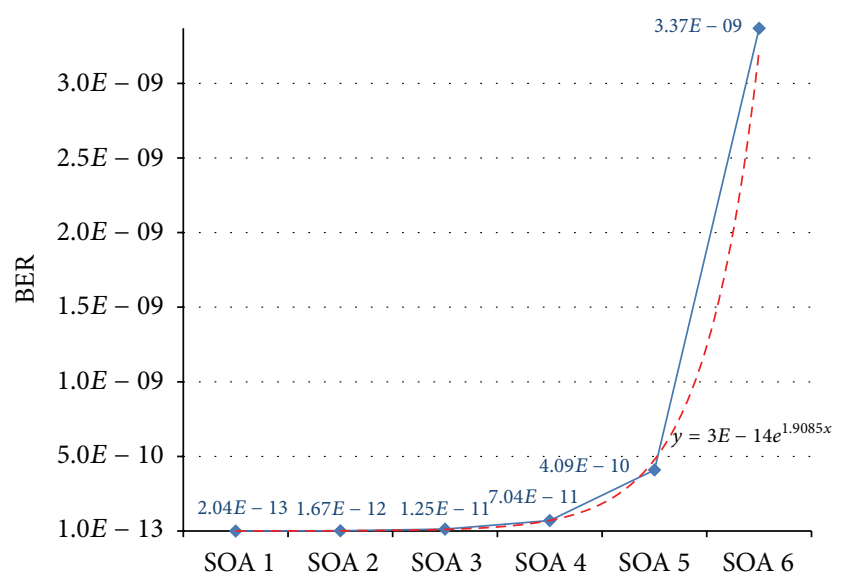

FIGURE 7: Average bit error rate (BER) versus number of cascaded SOAs.

and vertically after each stage. This is a consequence of increased jitter and distortion of the signal.

It is worth pointing out that the simulation model for the SOA, used in the tests described above, was calibrated with measurements coming from tests with real SOAs. This work was performed using a test system with a transmitter, a receiver, and a real SOA. The experimental setup used four wavelengths; each one of them carried data at $10 \mathrm{Gbps}$ using a pseudorandom binary sequence (PRBS) of length $2^{31}-1$ bits, without correlation among each other. At the receiver, we measured the BER for the four wavelengths. This experiment was performed first with a single stage and then with two SOAs in series to build a second stage.

4.4. Input Power Dynamic Range. For a large scale SOA switch fabric with cascaded SOAs, the output power of a certain SOA stage becomes the input power of the next SOA stage after a fixed passive loss. Therefore, the input power dynamic range (IPDR) match is very important for each SOA stage. An experimental IPDR curve for a Kamelian SOA for a single $1550 \mathrm{~nm}$ channel modulated at $10 \mathrm{~Gb} / \mathrm{s}$ with a PRBS of length $2^{15}-1$ was used as a reference for the simulation model. The characteristic " $U$ " shape of the power dynamic range of an SOA is the result of the presence of amplified spontaneous emission (ASE) at low optical input power and patterning distortion at high optical input power.

The VPI simulation model was also used to determine the IPDR curves of cascaded SOAs. The model did not consider signal regeneration in order to study a system as simple as possible. Figure 9 shows the IPDR simulation results in terms of the average power penalty for cascaded SOAs with ten wavelengths. The power penalty produced by the increase of cascaded SOAs would limit the input power range as it is also illustrated in the same figure. For example, the IPDR is narrower for five cascaded SOAs than that for three cascaded SOAs.

4.5. Other Phenomena. The simulation model also considers the effects of linear and nonlinear optical factors due to chromatic dispersion, Kerr nonlinearity, and Raman, Brillouin, 


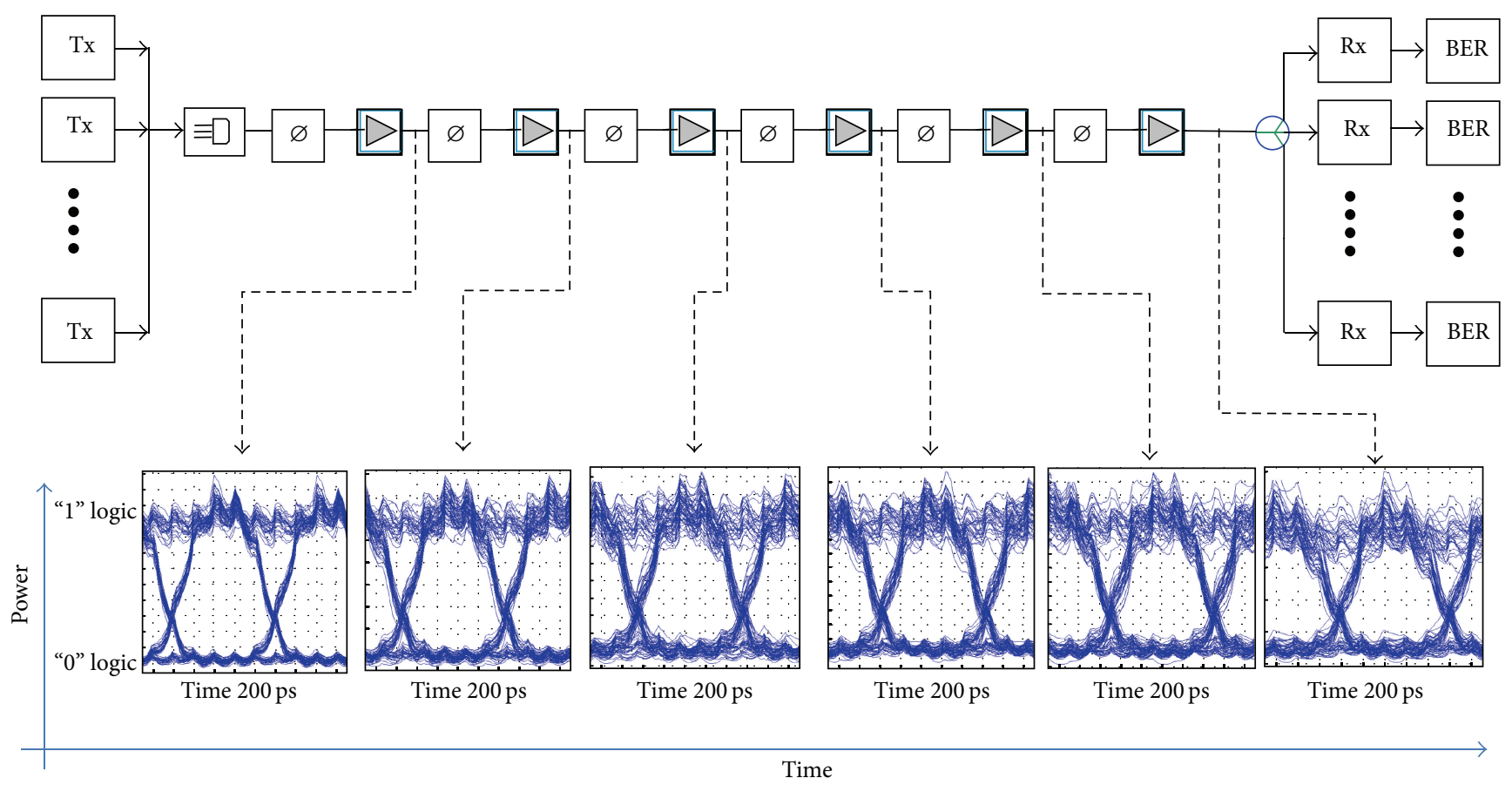

FIgURE 8: Cascading degradation simulated for 10 wavelengths.

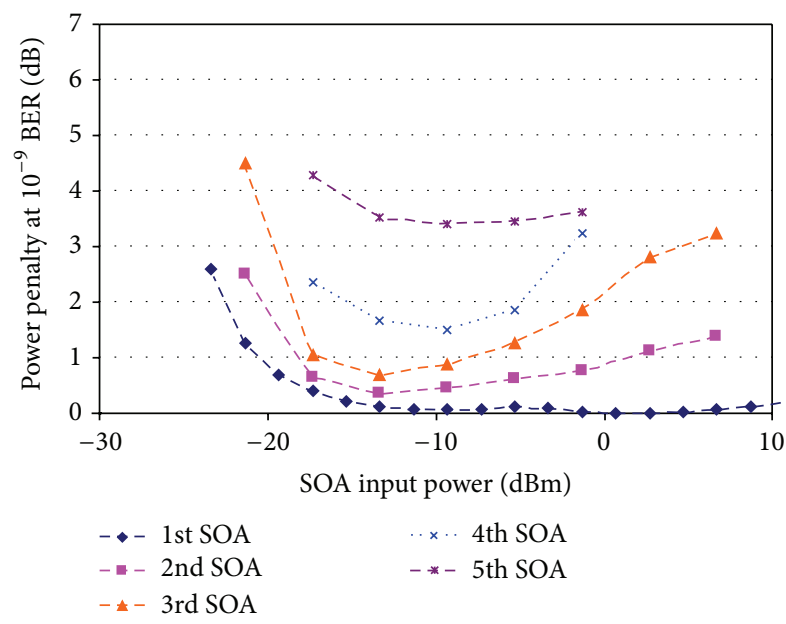

FIGURE 9: Input power dynamic range cascaded SOAs, VPI simulation.

and Rayleigh scattering. Noise penalties are also induced in the simulation model. The model also considers statistical independent noise, that is, shot noise and thermal noise. These are created with the aid of a random noise generator which is part of the VPItransmissionMaker simulator [16].

4.6. Simulation Model: Implementation and Calibration. We obtained the simulation results shown in this work by using the "VPI Photonics" software [16] in combination with a program written in Java to generate TCP traffic. We considered a design with ten wavelengths $(M=10$ in (1)), where each wavelength carries data at a rate of $10 \mathrm{~Gb} / \mathrm{s}$ using
$64 \mathrm{~B} / 66 \mathrm{~B}$ coding. The $64 \mathrm{~B} / 66 \mathrm{~B}$ scrambling, which is one-toone mapping of the data stream, provides data whitening. Such data whitening is intended to spread out erroneous data in the transmission [19]. The wavelengths were uniformly distributed from $\lambda_{1}=1550.71 \mathrm{~nm}$ to $\lambda_{10}=1554.33 \mathrm{~nm}$ and were spaced $0.4 \mathrm{~nm}(50 \mathrm{GHz})$ apart.

One of the key operating concerns in the simulations was to ensure linear operation. To this end, the input power levels were monitored in order to determine whether or not the device was driven into saturation. This issue is crucial in multiwavelength applications where a band of channels is amplified. The appropriate input power was determined by performing several VPI simulations.

Phenomena such as spontaneous emission, stimulated absorption, and stimulated emission were also taken into consideration and incorporated in the model by means of semiconductor rate equations. As previously mentioned, a number of factors were also included in the SOA simulation model implemented with VPI Photonics, which was calibrated with experimental data. In this way, the simulation model was tuned using a heuristic approach in order to operate as closely as possible to commercially available SOA devices, which are intended to be used in laboratory tests. The experimental data that were used to validate the model were reported in [20].

4.7. Considerations for the Analysis. The basic switching system architecture previously described in Section 2 was considered as the basic framework for the performance comparison of the different network topologies. The analysis considers a scenario with the following characteristics:

(i) Use of the high bandwidth that optical links can offer. 
(ii) Absence of electrooptical conversion of the signal in middle stages.

(iii) Low latency, which results from the use of multiple wavelengths and fast-striped switching.

(iv) The links that are considered to be short interconnections, that is, approximately 10 meters.

In addition, the analysis considers switching structures with full connectivity between nodes. Such structures are considered to be wide-sense and nonblocking in order to calculate the number of devices required for the tree topology. The couplers that were considered were $2 \times 2$ couplers. The number of couplers for the tree topology was calculated using a crossbar switch as a reference. The required number of couplers takes into consideration the number of inputs and outputs in order to interconnect all nodes $(N)$ and, thus, it is $N \times N$. The crossbar switch includes SOAs in each connection to broadcast and select the path. The cascadability study considers the number of SOAs in a row as a cascading parameter. Nevertheless, the cascaded SOAs can be used in other switches such as Clos, Spanke, and Benes [2].

\section{Results and Discussion}

The quantitative analysis is divided into several parts: the analysis related to the cascading properties of the network topologies, the scaling characteristics, and the evaluation of the latency generated by transmission and propagation times. In addition, the quantitative analysis related to the scaling properties considers two characteristics: the increment in the number of devices and the scalability cost assigned by the cost-weight parameters, as suggested in $[21,22]$.

5.1. Cascading Results. The number of cascaded SOAs is a significant parameter to consider for the selection of a network topology. Figure 10 shows how the number of cascaded SOAs increases when the number of interconnected nodes increases. We show results for a single ring and for a double ring (i.e., a ring for clockwise transmissions and another for the opposite direction).

Each topology requires a different number of cascading SOAs to connect nodes and this number determines the BER. Figure 11 shows the BER for the topologies under investigation as a function of cascaded SOAs and couplers. As it can be seen, the tree and single ring topologies quickly reach the cascadability limit previously discussed, when the number of interconnected nodes increases.

For all studied topologies, there are excess losses between cascaded SOAs due to the passive components in the system. In addition, each cascaded stage also introduces the same attenuation. The losses are estimated to be around $9 \mathrm{~dB}$ at each stage. These factors are studied in more detail in the following section.

5.2. Scaling Results. The required number of components determines which topology is more scalable when the number of nodes is incremented. The most relevant components for this analysis are the SOAs and the couplers. This is due to

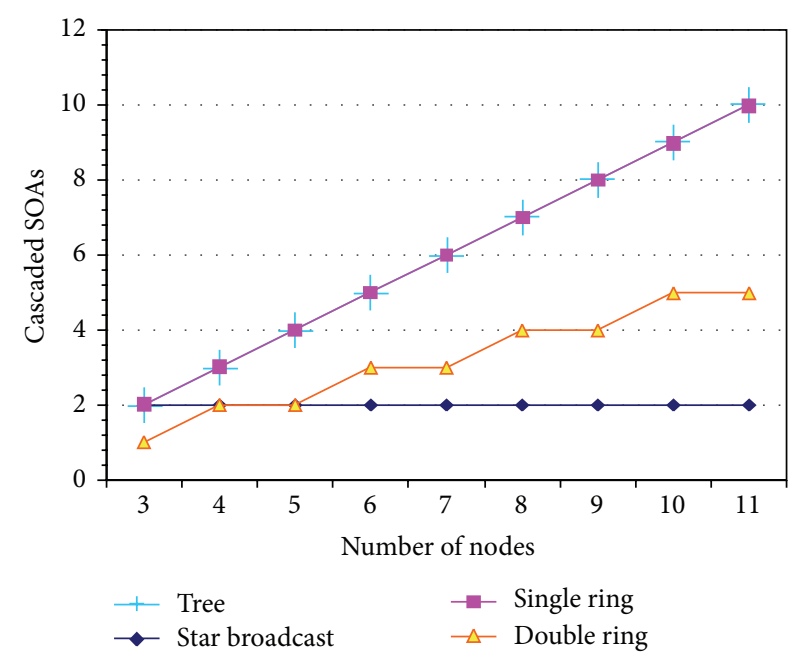

FIgURE 10: Number of cascaded SOAs versus number of nodes.

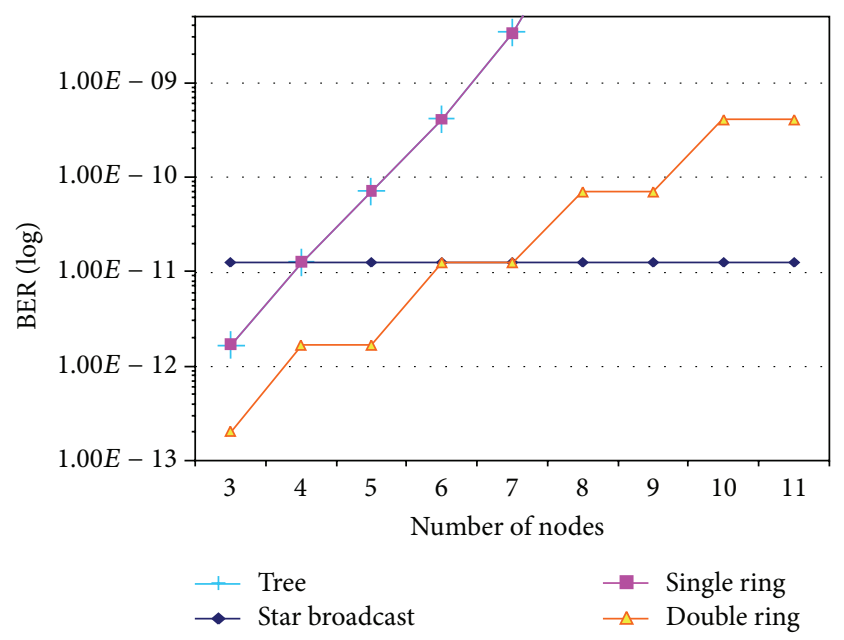

FIGURE 11: Bit error rate (BER) versus number of nodes for the topologies studied.

the fact that they are the components that differ in number from topology to topology. In contrast, there is the same number of lasers and array wavelength gates (AWGs) for all topologies. For instance, if a transmission takes place between two nodes, then the same numbers of transmitters and receivers (i.e., wavelengths) are used in the system for each one of the studied topologies.

Figure 12 shows the number of couplers needed to increase the number of nodes in each one of the considered topologies. In this case, the worst situation happens in the star and tree topologies because the number of couplers increases more rapidly when the number of nodes is increased. This increase in the number of couplers affects the signal-to-noise ratio and, thus, a degradation of the signal is introduced. The single ring topology uses the least number of couplers, as it is also shown in Figure 12.

Figure 13 illustrates that the broadcast star uses the least number of SOAs per node, followed by the single ring and the double ring. And, finally, the tree topology utilizes more 


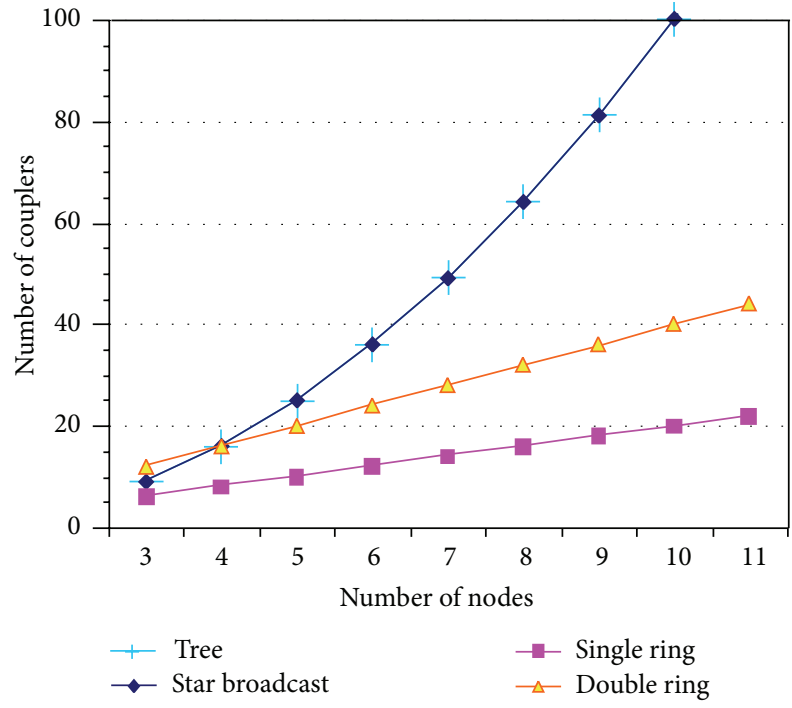

FigURE 12: Number of required couplers versus number of nodes.

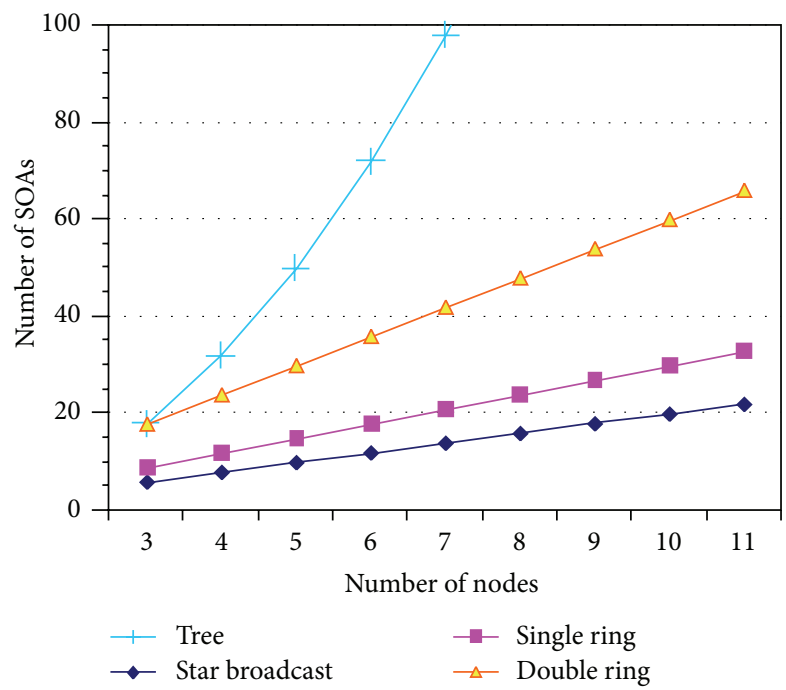

FIGURE 13: Number of required SOAs versus number of nodes.

SOAs and, therefore, it needs more components. This is in order to have full interconnection between nodes and to be able to control the desired communication path.

In order to study the scaling properties of different network topologies, we take into consideration the level of signal degradation that arises when the number of nodes is increased.

The signal attenuation due to the network topology can be determined by the sum of the losses in the communication medium, the devices, and the couplers. The losses in the fourport directional coupler as defined by Palais [23] are throughput $\left(L_{\mathrm{THP}}\right)$, tap $\left(L_{\mathrm{TAP}}\right)$, directionality $\left(L_{\mathrm{DIR}}\right)$, and excess $\left(L_{E}\right)$ losses. In particular, coupler losses are significant for various scalable applications. The estimation of the coupling loss is significant in calculating differences among topologies since each structure requires a different number of couplers, which results in different power losses and different levels of signal degradation.

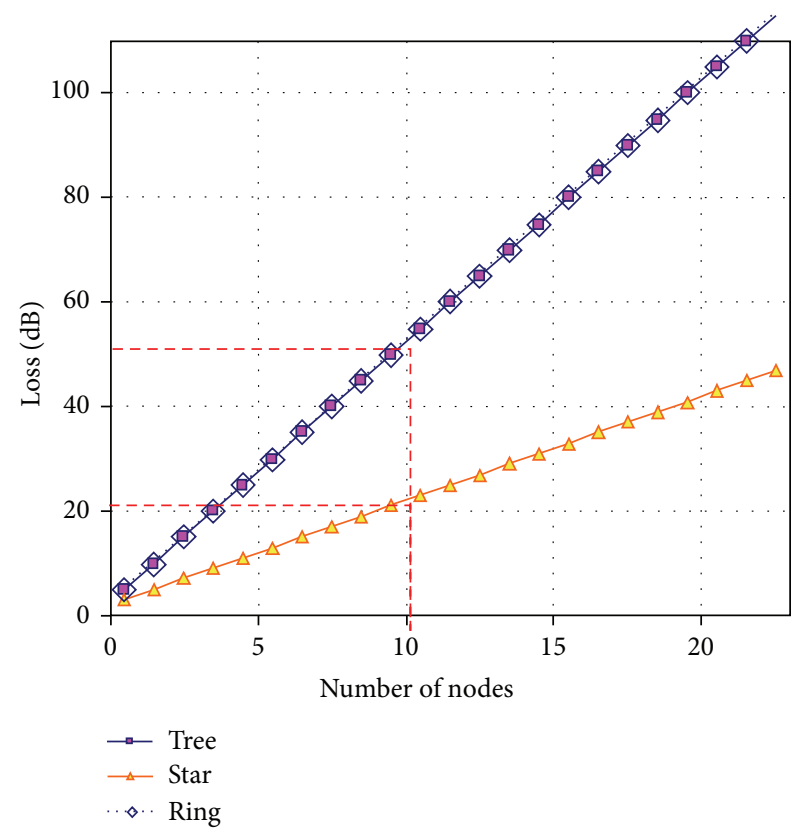

FIgURE 14: Number of nodes versus coupler losses.

The coupler losses were calculated for each topology as follows. The different types of losses were calculated taking into consideration the coupler ports. These losses are characterized by the following equations [23]. For the tree and ring topologies, the coupler losses $(L)$ are given by

$$
L=(N-1) L_{\mathrm{THP}}+L_{\mathrm{TAP}}+2 N L_{C}
$$

where $N$ is the number of nodes and $L_{C}$ is the loss per connection in the couplers (set to $1 \mathrm{~dB}$ in the calculations). Parameters $L_{\text {THP }}$ and $L_{\text {TAP }}$ are also measured in decibels.

For the star topology, the coupler losses are

$$
L=-10 \log \left(\frac{1}{N}\right)+L_{E}+2 L_{C} .
$$

Figure 14 shows the results of the coupler losses for the three considered topologies. As seen in this figure, the coupler losses linearly increase with the number of network nodes for the three topologies. But, whereas the tree and ring topologies exhibit similar losses, the star topology shows less degradation. For example, 10 nodes cause a coupler loss of approximately $20 \mathrm{~dB}$ in the star, whereas the coupler losses in the ring and tree are approximately $50 \mathrm{~dB}$. This result shows that network scaling in the ring and tree topologies has severe consequences in power loss and, hence, the power budget requires a careful design. In terms of coupler loss, therefore, the star topology is seen to be more efficient. Nevertheless, in the central distributor of the star, the coupler loss is significant.

5.3. Latency and Its Effect on TCP Performance. The latency of the wavelength-striped system is crucial for the performance of data transmission. This analysis assumes that the propagation time between nodes is significantly smaller than the 


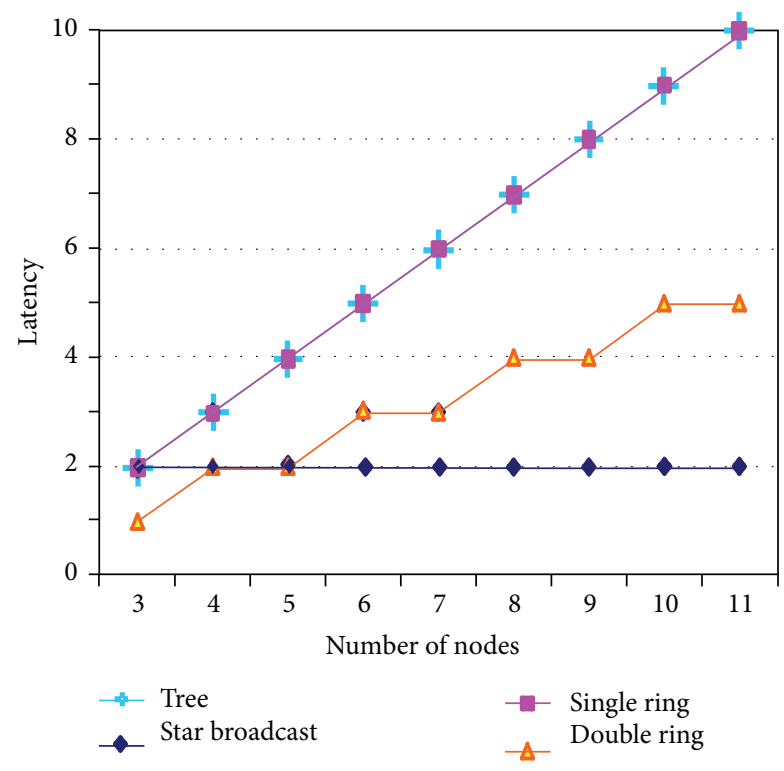

FIGURE 15: Latency units versus number of nodes.

transmission time. As previously mentioned, in this work, we consider short-reach optical interconnects with a distance between nodes set to $10 \mathrm{~m}$ which corresponds to a $50 \mathrm{~ns}$ delay for the propagation time over fiber with a refractive index of 1.5. The transmission time was related to the time it takes to transmit 1 Mbyte of data at a rate of $10 \mathrm{~Gb} / \mathrm{s}$. This way, the total latency between end-nodes comes from these transmission and propagation times. The transmission time is defined as the time it takes for a transmitter to release a block of bits (i.e., a data frame) to the medium. The propagation time is defined as the time it takes for a bit to travel over the link from the source to the destination. The evaluation of the latency in the system was normalized by considering that a unit of latency equals the latency between two nodes. Thus, one time unit was assigned for the latency, that is, transmission plus propagation times between adjacent nodes, in the tree and ring topologies. Two time units were assigned for the star topology: one time unit represents the time taken to travel from the source node to the central distributor and the other time unit is the trip from the central distributor to the destination node. Figure 15 shows the latency results shown in normalized latency units.

The effects of latency were tested with TCP traffic injected to the simulation. TCP creates a connection for each transfer and then sends data fragments in segments. This approach combines connection-oriented communication and packetswitching.

There is a closed-loop feedback inferred by TCP from the flow of segments from the sender to the receiver. This flow could be expressed by the goodput of the transmission. The goodput is the amount of data successfully received per time unit at the receive end and can be expressed as follows:

$$
\text { goodput }=\text { throughput }- \text { overhead }- \text { rollbacks. }
$$

Throughput, that is, the amount of data per time unit transferred by the network, and possibly passing through a

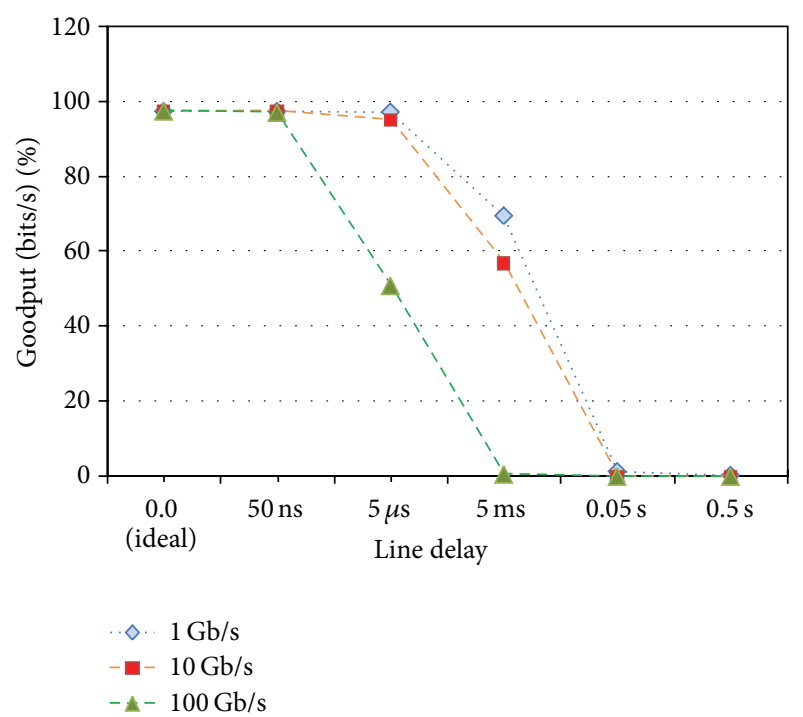

Figure 16: Goodput versus line delay for a BER of $10^{-9}$.

particular point in a network system, is expressed as “ $x$ ” b/s. Overhead is expressed as " $y$ " bits divided by the processing time for the TCP, IP, and Ethernet headers. Rollbacks are expressed as " $z$ " bits divided by the acknowledgment trip time and/or the retransmission time when a packet is lost during the trip or when bits with errors arrive at the destination node. These errors may be a consequence of noise or packet loss during transmission.

Latency negatively affects TCP because the growth of its window size depends on the round trip time and, thus, the goodput is also affected. For these reasons, it is deduced that TCP/IP operates advantageously in short optical interconnects (i.e., in the order of nanosecond delays), but not in longdistance links, even though TCP/IP may maintain a reliable communication.

The TCP window is the number of segments that have been transmitted, but not acknowledged at a given time. TCP provides reliable connection because it confirms that packets are correctly received by the use of acknowledgements. The sending rate of each TCP transmission shows a dynamic behavior and varies according to the number of received acknowledgments. In case of packet loss or congestion, the transmission rate is decreased by the sender. These variations take the form of an additive increase and multiplicative decrease, known as AIMD flow.

The fundamental behavior of TCP is independent of the topology of the network. However, factors such as latency and flow rate control can considerably change the output of the system. Selected client-server connections using TCP were simulated using SSFNet Java simulator and some experiments were carried out to illustrate the effects of latency on goodput. Figure 16 shows these results for three different data rates. The case with $100 \mathrm{~Gb} / \mathrm{s}$ clearly shows the effect of delay on goodput, which dramatically decreases from $97 \%$ to $50 \%$, when the latency of the link was increased from $50 \mathrm{~ns}$ to $5 \mu \mathrm{s}$. By taking these results into consideration, it can be observed that the goodput could be severely affected when the distance between each pair of the five cascaded SOAs is increased. 


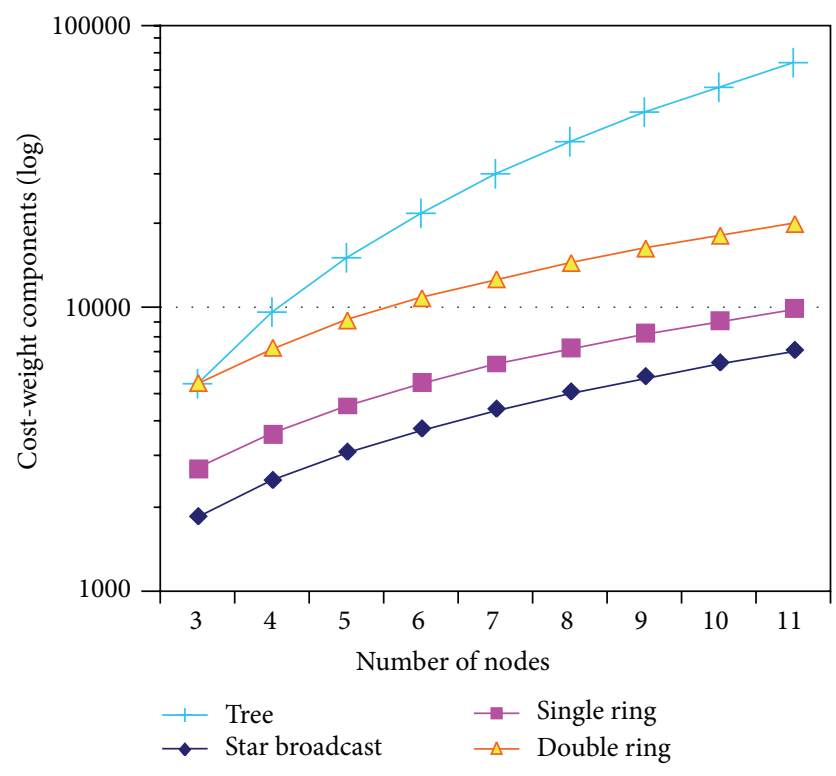

FIGURE 17: Cost-weight versus number of nodes.

Aggregated throughput is affected with distance due to the increased propagation delay and, as a consequence, when the round trip time of the TCP segments increases, the goodput is adversely affected. If the bit rate is also increased, the goodput is also affected by the enlarged link delay in presence of packet loss [24]. This is because, with higher bit rates, more packets are in transit and, therefore, more of them are lost when errors occur.

5.4. Cost-Weight Analysis. The outcome for the cost-weight analysis of the topologies, including couplers and SOAs, is depicted in Figure 17. The cost-weight is a relative price based on the ratio of the commercial prices of the components available in the market at the moment of this study.

The cost-weight average price reflects the essential costs in the implementation of each topology. For example, if the cost-weight for an SOA is calculated as 300, the one corresponding to AWGs is calculated as 40, which is the ratio adopted from [21]. In this analysis, it was chosen to use a coupler cost-weight value of 4 based on [22]. The cost-weight analysis is valid at the time of this writing, but it can change if technology prices change. Nevertheless, the cost ratios are shown as a reference to estimate the cost-weight shown in Figure 17.

A remark is that topologies which use fewer couplers do not represent a significant advantage because the cost-weight of the coupler is lower than the cost-weight of the SOA.

5.5. Analysis. In this subsection, some results are summarized and divided into four categories: cascadability and scalability, reliability, performance, and cost-weight.

5.5.1. Cascadability and Scalability. Fewer SOAs are used in the star topology and it is not necessary to increase the number of cascaded SOAs in order to have more connected nodes.
The scalability is directly related to the BER which significantly increases for each cascaded SOA. The star configuration needs fewer cascaded SOAs and, consequently, a larger network can be implemented. However, the central distributor requires significant increases in the number of couplers in order to scale.

5.5.2. Reliability. With the passive ring topology using bypass links, if a node fails, only that node is affected. If the node regenerates the signal in the ring without bypass, the entire system shuts down when a node fails.

In the star topology, the central distributor is crucial. If problems occur in this component, then the entire network fails. In the ring, as well as in the tree topology, a defective shared medium may also result in failure of the entire network.

5.5.3. Performance. Latency reduction is a key factor in achieving very high bit rates and it is particularly important for a feedback loop system, for example, TCP/IP. The traffic carried by a star topology only has to travel from the node to the central distributor and from the central distributor to the node (two cascading stages). Therefore, in most cases, the star topology has the lowest latency if equal delay units between nodes are considered. This is an important factor in coping with latency.

5.5.4. Cost-Weight. The single ring and the star topologies have the best cost-weight because they use cheaper components and, consequently, offer potential advantages in commercial applications. We emphasize that this result considers the cost ratios at the moment of the analysis and can vary as costs change.

\section{Conclusions}

In this work, three topological alternatives to the architecture of a wavelength striping electrooptical switch have been analyzed. In particular, the cascadability of different topologies of the system has been studied and bit error rate parameters were calculated up to $6 \mathrm{SOA}$ stages. The evaluation of the tree, ring, and star structures shows that the star topology presents the lowest latency. Furthermore, the results of the analysis and BER measurements show that in general the star topology offers more advantages than the other topologies. The proposed star also has better costweight and latency figures, which significantly affects the performance of the system. This is so because even when the number of nodes is increased, the transmission distance is always measured from the node to the central distributor. The proposed star topology can better scale to more nodes than the other approaches. As a consequence, it can help to reduce the latency, which represents a valuable advantage when a feedback-loop protocol, such as TCP/IP, is used.

Finally, we emphasize that this work has illustrated the need of improving protocol and hardware design, and for the further evolution of electrooptical interconnects. For instance, in a LAN, higher levels of bit error rate and 
packet error rate would be expected because of the increased distance between nodes. Therefore, in order to apply the wavelength striping approach, new solutions are required for problems such as signal dispersion, power budget, and medium access control (MAC). In addition, more robust coding schemes and routing control planes have to be designed.

\section{Conflict of Interests}

The authors declare that there is no conflict of interests regarding the publication of this paper.

\section{Acknowledgments}

The first author was supported by a scholarship granted by the National Council of Science and Technology of Mexico (CONACyT). He also thanks Professors R. V. Penty and I. H. White from University of Cambridge, Photonics Group, for their suggestions and valuable advice in the early stages of this work.

\section{References}

[1] K. E. Zoiros, A. Kalaitzi, and C. S. Koukourlis, "Study on the cascadability of a SOA-assisted Sagnac switch pair," Optik, vol. 121, no. 13, pp. 1180-1193, 2010.

[2] I. H. White, K. A. Williams, R. V. Penty et al., "Control architecture for high capacity multistage photonic switch circuits," Journal of Optical Networking, vol. 6, no. 2, pp. 180-188, 2007.

[3] F. Ayadi, J. F. Hayes, and M. Kavehrad, "WDM cross-connected star topology for the bilayered ShuffleNet," Journal of Lightwave Technology, vol. 12, no. 9, pp. 1668-1678, 1994.

[4] M. Barranco, J. Proenza, G. Rodriguez-Navas, and L. Almeida, "An active star topology for improving fault confinement in CAN networks," IEEE Transactions on Industrial Informatics, vol. 2, no. 2, pp. 78-85, 2006.

[5] P. W. Dowd, "Random access protocols for high-speed interprocessor communication based on an optical passive star topology," Journal of Lightwave Technology, vol. 9, no. 6, pp. 799$808,1991$.

[6] N. Mehravari, "Performance and protocol improvements for very high speed optical fiber local area networks using a passive star topology," Journal of Lightwave Technology, vol. 8, no. 4, pp. 520-530, 1990.

[7] N. Nadarajah, "Control packet signaling mechanism using electronic code division multiple access for packet-based networks," Optics Communications, vol. 281, no. 19, pp. 4956-4970, 2008.

[8] A. Narula-Tam, E. Modiano, and A. Brzezinski, "Physical architecture design for survivable routing of logical rings in WDM-based networks," in Proceedings of the IEEE Global Communications Conference (GLOBECOM '03), vol. 5, pp. 2552-2557, San Francisco, Calif, USA, December 2003.

[9] M. A. Mohd and M. M. Ahmed, "Fiber optics: an antidote to electromagnetic interference (EMI)," in Southcon/94. Conference Record, p. 312, IEEE, Piscataway, NJ, USA, 1994.

[10] E. Rodríguez-Colina, L. B. James, R. V. Penty, I. H. White, K. A. Williams, and A. W. Moore, "TCP sending rate control at terabits per second," in Proceedings of the 25th IEEE International
Conference on Computer Communications (INFOCOM '06), pp. 1-5, IEEE, Barcelona, Spain, April 2006.

[11] T. Lin, K. A. Williams, R. V. Penty, I. H. White, and M. Glick, "Capacity scaling in a multihost wavelength-striped SOA-based switch fabric," Journal of Lightwave Technology, vol. 25, no. 3, pp. 655-663, 2007.

[12] E. Rodriguez-Colina, TCP/IP over cascaded SOA-based multiple wavelength-striped short reach router interconnect [Ph.D. thesis], University of Cambridge, Cambridge, UK, 2009.

[13] A. S. Tanenbaum, Computer Networks, Prentice-Hall, Upper Saddle River, NJ, USA, 2nd edition, 1995.

[14] J. Leuthold, R. Bonk, T. Vallaitis et al., "Linear and nonlinear semiconductor optical amplifiers," in Proceedings of the Conference on Optical Fiber Communication, Collocated National Fiber Optic Engineers Conference (OFC/NFOEC '10), pp. 1-3, IEEE, San Diego, Calif, USA, March 2010.

[15] S. Singh, A. Singh, and R. S. Kaler, "Performance evaluation of EDFA, RAMAN and SOA optical amplifier for WDM systems," Optik, vol. 124, no. 2, pp. 95-101, 2013.

[16] VPI photonics, December 2015, http://www.vpiphotonics.com.

[17] G. P. Agrawal and N. A. Olsson, "Self-phase modulation and spectral broadening of optical pulses in semiconductor laser amplifiers," IEEE Journal of Quantum Electronics, vol. 25, no. 11, pp. 2297-2306, 1989.

[18] D. D’Alessandro, G. Giuliani, and S. Donati, "Spectral gain and noise evaluation of SOA and SOA-based switch matrix," IEE Proceedings: Optoelectronics, vol. 148, no. 3, pp. 125-130, 2001.

[19] A. W. Moore, L. B. James, A. Wonfor et al., "Chasing errors through the network stack: a testbed for investigating errors in real traffic on optical networks," IEEE Communications Magazine, vol. 43, no. 8, pp. s34-s39, 2005.

[20] T. Lin, K. A. Williams, R. V. Penty, I. H. White, M. Glick, and D. McAuley, "Performance and scalability of a single-stage SOA switch for $10 \times 10 \mathrm{~Gb} / \mathrm{s}$ wavelength striped packet routing," IEEE Photonics Technology Letters, vol. 18, no. 5, pp. 691-693, 2006.

[21] R. Van Caenegem, D. Colle, M. Pickavet, and P. Demeester, "Scalable designs for all-optical packet-switching nodes," in Proceedings of the 9th International Conference on Transparent Optical Networks (ICTON '07), vol. 3, pp. 218-221, IEEE, Rome, Italy, July 2007.

[22] E. Zouganeli, A. Solem, H. Kjonsberg et al., "Techno-economic evaluation of optical packet/burst switching," in Proceedings of the 31st European Conference on Optical Communication (ECOC '05), vol. 4, pp. 967-968, IEEE, Glasgow, UK, September 2005.

[23] J. C. Palais, Fiber Optic Communications, Prentice-Hall, Englewood, NJ, USA, 4th edition, 1998.

[24] E. Rodriguez-Colina, A. W. Moore, M. Glick, A. Wonfor, I. H. White, and R. V. Penty, "BER implication for TCP/IP network throughput over a $10 \times 10$ Gbps Wavelength-Striping Cascaded SOA-Switch," in Proceedings of the London Communications Symposium, London, UK, 2006. 


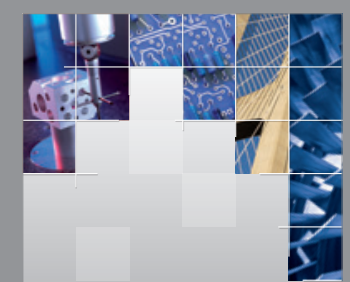

\section{Enfincering}
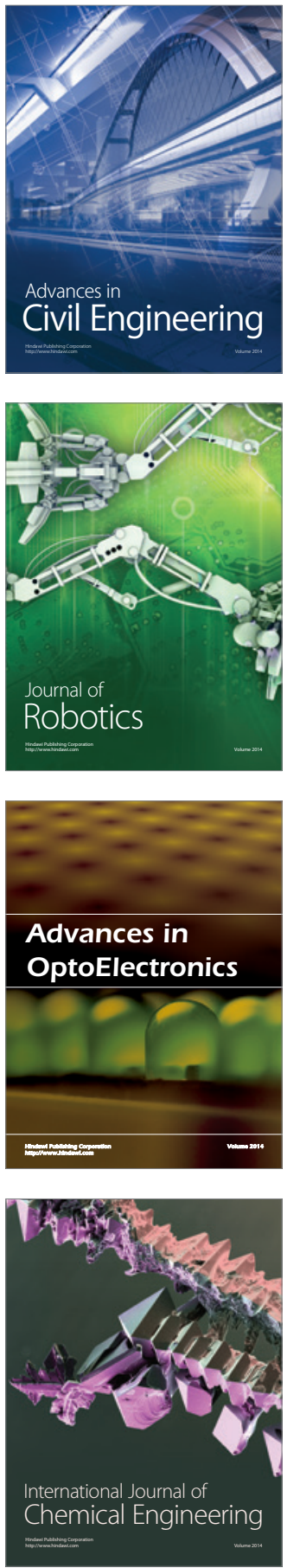

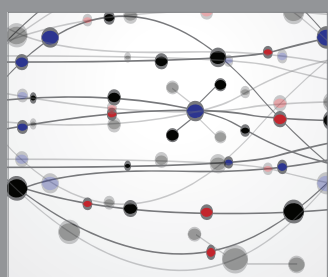

The Scientific World Journal

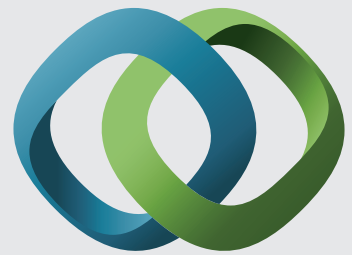

\section{Hindawi}

Submit your manuscripts at

http://www.hindawi.com
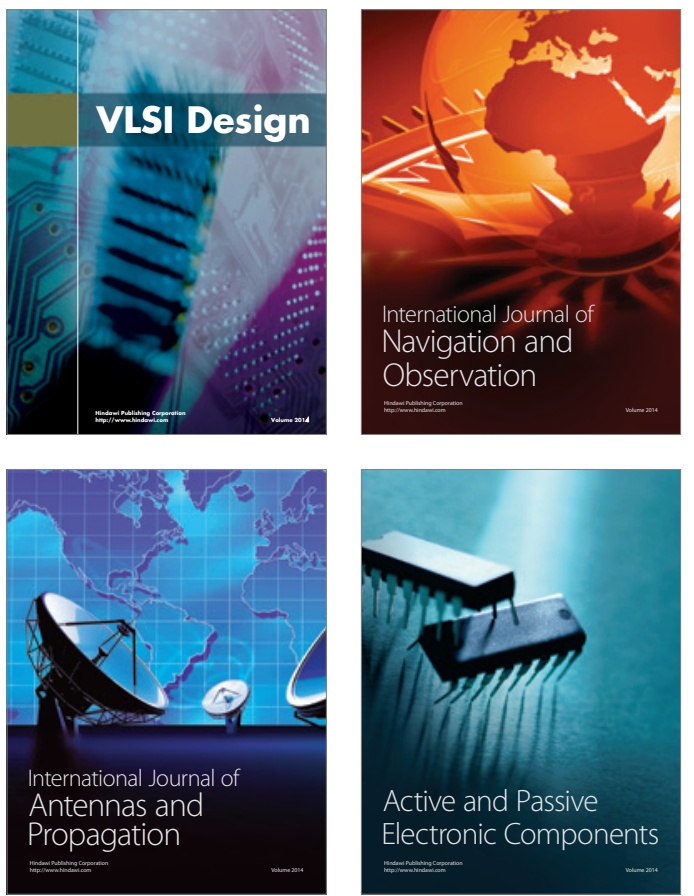
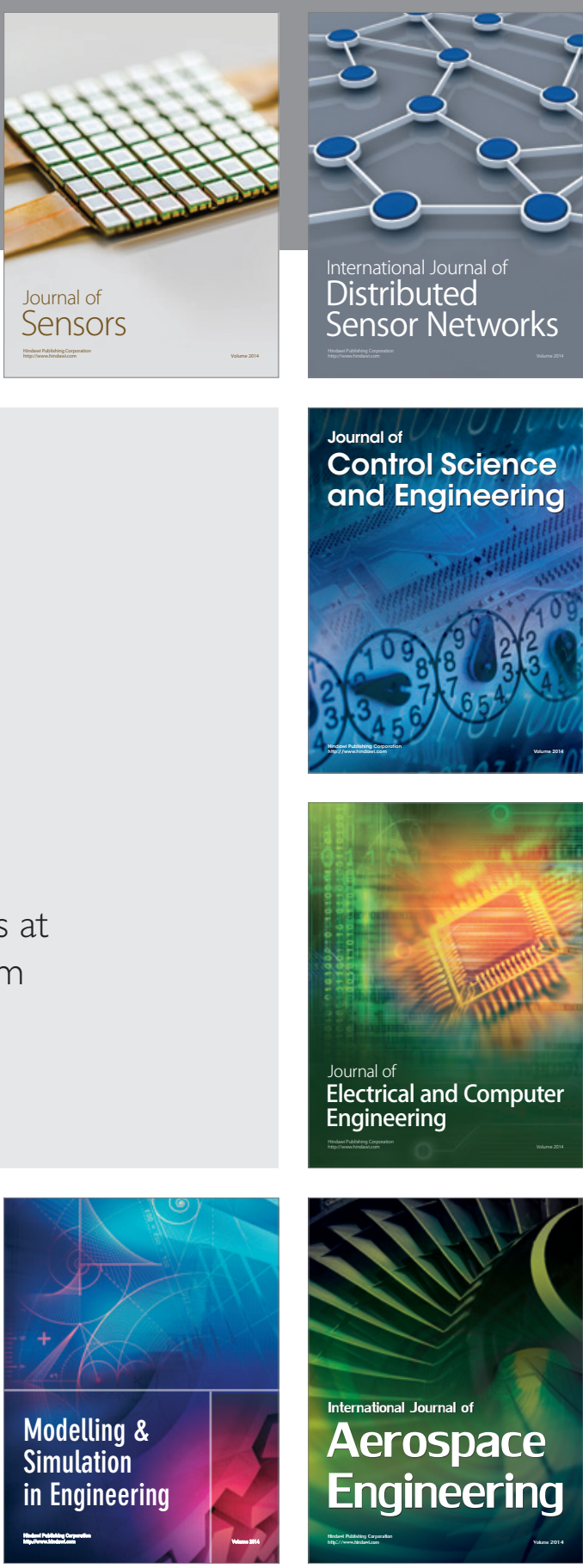

International Journal of

Distributed

Sensor Networks

Journal of

Control Science

and Engineering
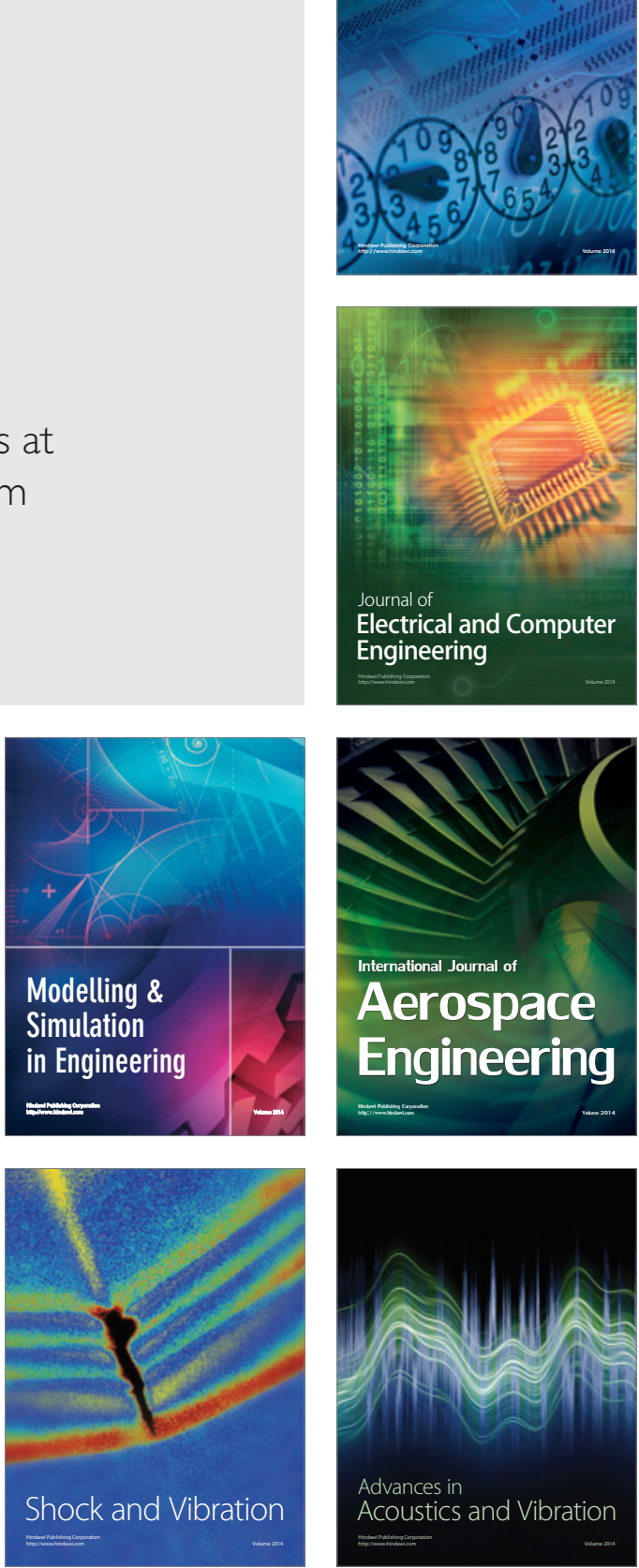